\title{
A comparative analysis of library prep approaches for sequencing low input translatome samples
}

Yang Song ${ }^{1}$, Beatrice Milon², Sandra Ott ${ }^{1}$, Xuechu Zhao ${ }^{1}$, Lisa Sadzewicz ${ }^{1}$, Amol Shetty ${ }^{1}$, Erich T. Boger ${ }^{3}$, Luke J. Tallon', Robert J. Morell ${ }^{3}$, Anup Mahurkar ${ }^{1}$ and Ronna Hertzano ${ }^{1,2,4^{*}}$

\begin{abstract}
Background: Cell type-specific ribosome-pulldown has become an increasingly popular method for analysis of gene expression. It allows for expression analysis from intact tissues and monitoring of protein synthesis in vivo. However, while its utility has been assessed, technical aspects related to sequencing of these samples, often starting with a smaller amount of RNA, have not been reported. In this study, we evaluated the performance of five library prep protocols for ribosome-associated mRNAs when only 250 pg-4 ng of total RNA are used.

Results: We obtained total and RiboTag-IP RNA, in three biological replicates. We compared 5 methods of library preparation for Illumina Next Generation sequencing: NuGEN Ovation RNA-Seq system V2 Kit, TaKaRa SMARTer Stranded Total RNA-Seq Kit, TaKaRa SMART-Seq v4 Ultra Low Input RNA Kit, Illumina TruSeq RNA Library Prep Kit V2 and NEBNext ${ }^{\oplus}$ Ultra ${ }^{\text {TM }}$ Directional RNA Library Prep Kit using slightly modified protocols each with 4 ng of total RNA. An additional set of samples was processed using the TruSeq kit with $70 \mathrm{ng}$, as a 'gold standard' control and the SMART-Seq v4 with $250 \mathrm{pg}$ of total RNA. TruSeq-processed samples had the best metrics overall, with similar results for the $4 \mathrm{ng}$ and $70 \mathrm{ng}$ samples. The results of the SMART-Seq v4 processed samples were similar to TruSeq (Spearman correlation >0.8) despite using lower amount of input RNA. All RiboTag-IP samples had an increase in the intronic reads compared with the corresponding whole tissue, suggesting that the IP captures some immature mRNAs. The SMARTer-processed samples had a higher representation of ribosomal and non-coding RNAs leading to lower representation of protein coding mRNA. The enrichment or depletion of IP samples compared to corresponding input RNA was similar across all kits except for SMARTer kit.
\end{abstract}

Conclusion: RiboTag-seq can be performed successfully with as little as $250 \mathrm{pg}$ of total RNA when using the SMART-Seq v4 kit and $4 \mathrm{ng}$ when using the modified protocols of other library preparation kits. The SMART-Seq v4 and TruSeq kits resulted in the highest quality libraries. RiboTag IP RNA contains some immature transcripts.

Keywords: RiboTag, Library preparation kits, Low-input RNA-seq, RNA-seq, Coverage bias

\footnotetext{
* Correspondence: rhertzano@som.umaryland.edu

${ }^{1}$ Institute for Genome Sciences, University of Maryland School of Medicine,

Baltimore, MD 21201, USA

2Department of Otorhinolaryngology-Head \& Neck Surgery, University of

Maryland School of Medicine, Baltimore, MD 21201, USA

Full list of author information is available at the end of the article
}

(c) The Author(s). 2018 Open Access This article is distributed under the terms of the Creative Commons Attribution 4.0 International License (http://creativecommons.org/licenses/by/4.0/), which permits unrestricted use, distribution, and reproduction in any medium, provided you give appropriate credit to the original author(s) and the source, provide a link to the Creative Commons license, and indicate if changes were made. The Creative Commons Public Domain Dedication waiver (http://creativecommons.org/publicdomain/zero/1.0/) applies to the data made available in this article, unless otherwise stated. 


\section{Background}

Considerable scientific effort has been dedicated to understanding cell type-specific expression profiles from complex tissues, such as brain, liver, pancreas, testes, eye or ear [1-7]. To overcome the issue of cellular heterogeneity within complex tissues, two methods have been traditionally used in mice: Laser-Capture Microdissection (LCM) $[8,9]$ and Fluorescence Activated Cell Sorting (FACS) $[10,11]$. However, LCM is a laborious and time-consuming procedure with low yield of mRNA; and FACS requires tissue dissociation - which may lead to changes in gene expression - and requires dedicated equipment [12]. More recently, single cell RNA-seq has been introduced. However, this technique too requires tissue dissociation and is currently limited by the number of genes detected per sequenced cell $[13,14]$. To overcome these limitations, Translating Ribosome Affinity Purification (TRAP) [15] and RiboTag [16] have been recently developed to study cell type-specific transcriptome profiles. Both methods rely on immunoprecipitation of ribosome-attached RNA (also named 'translatome') by cell type-specific molecular targeting of the ribosomal proteins, often in a Cre-lox based system [15-17]. These methods have the advantage of not requiring tissue dissociation, thus allowing for cell type-specific translatome analysis from intact tissues.

While ribosome-attached RNA sequencing for expression analysis has been validated from a biological standpoint $[18,19]$, the technical aspects of its library construction and sequencing have not been studied. In instances where small complex tissues are studied, the amount of starting material after immunoprecipitation may be limited (e.g., less than $5 \mathrm{ng}$ ). When starting from low amounts of RNA, additional cycles of amplification using PCR are performed after adapter ligation to amplify the cDNA to generate enough material for sequencing. Multiple commercial kits are available in the market to build cDNA libraries from samples with low amounts of RNA, including kits from NuGEN, New England Biolabs (NEB), Illumina and TaKaRa. Standard protocols for library construction are commonly designed to start with more than $100 \mathrm{ng}$ of total RNA [20, 21] and only a few studies have been conducted to compare the performance of library preparation kits using less than $5 \mathrm{ng}$ of total RNA as their starting amount $[22,23]$. In this study, we selected four of the commonly used library preparation kits that are also suitable for lower-input samples for comparison. We modified the standard protocols for NEB and Illumina library preparation kits to enable them to work with smaller amounts of RNA than the recommended amounts down to as little as $4 \mathrm{ng}$ of total RNA. We included one kit, SMART-Seq v4, that was designed for single cell RNA-seq and tested it with $4 \mathrm{ng}$ and $250 \mathrm{pg}$ of total RNA. We evaluated the performance of the different kits based on duplication rate, percentage of intronic and exonic regions being detected, the evenness of coverage of transcripts and ribosomal RNA read-count in comparison to total reads. We also compared the reproducibility of the enrichment or depletion effect based on ribotag-translatome profile for the first time.

\section{Methods \\ Animals}

The Gfi1-Cre knock-in mice generated by Dr. Lin Gan (University of Rochester) were kindly provided by Dr. Jian Zuo of the Developmental Neurobiology Department at St. Jude Children's Research Hospital. RiboTag mice generated by Dr. Paul S Amieux (University of Washington) were kindly provided by Dr. Mary-Kay Lobo of the Department of Neurobiology and Anatomy at University of Maryland Baltimore. B6.Cg-Gt(ROSA)26-

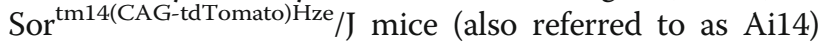
were purchased from the Jackson Laboratory (stock \#007914, Bar Harbor, ME). Experimental animals for RNA-seq, Gfi1 ${ }^{\mathrm{Cre} /{ }^{+}} ;$RiboTag $^{\mathrm{HA} / \mathrm{HA}}$, were obtained by crossing RiboTag mice with Gfi1-Cre mice. Animals for immunostaining, Gfi1 ${ }^{\mathrm{Cre} /{ }^{+}}$;Ai14, were obtained by crossing Gfi1-Cre mice with Ai14 mice [24]. All procedures involving animals were carried out in accordance with the National Institutes of Health Guide for the Care and Use of Laboratory Animals and have been approved by the Institutional Animal Care and Use Committee at the University of Maryland, Baltimore (protocol numbers 1015003 and 0915006).

\section{Ribosome immunoprecipitation and RNA extraction}

Three 30-day old Gfi1 ${ }^{\mathrm{Cre} /+}$;RiboTag ${ }^{\mathrm{HA} / \mathrm{HA}}$ mice were euthanized by $\mathrm{CO}_{2}$ asphyxiation followed by cervical dislocation. Livers were harvested and immediately frozen on dry ice. Equal amounts of liver were used for input RNA extractions (RNeasy Plus Micro kit, QIAGEN USA, Germantown, MD, USA) or futher processed for ribosome immunoprecipitation $(5 \mu \mathrm{g}$ of purified antiHA.11, BioLegend, San Diego, CA, USA) followed by RNA extraction as previously decribed in Sanz et al., 2009 [16]. The RNeasy Plus Micro kit is optimized for the removal of genomic DNA through a combination of high salt buffer and the gDNA Eliminator spin column. Quality of the RNA was assessed on an Agilent Technologies Bioanalyzer 2100 RNA pico chip as per the manufacturer's instructions (Agilent Technologies, Palo Alto, CA, USA). All samples had a RIN score of 10 and no evidence of DNA contamination in the form of a high molecular weight DNA band. All RNA was equally aliquoted to test for the performance of five commercial kits and seven protocols. 


\section{Real-time RT-PCR}

Efficiency of the ribosome immunoprecipitation was assessed by reverse transcription followed by real time PCR. One nanogram of total RNA from the input and the IP samples was used for reverse transcription using the Maxima First Strand cDNA Synthesis Kit for RT-qPCR (Thermo Fisher Scientific, Waltham, MA, USA). The real time PCR was performed on an Applied Biosystems ${ }^{\circ}$ StepOnePlus $^{\mathrm{TM}}$ Real-Time PCR System with the Maxima SYBR Green/ROX qPCR Master Mix (Thermo Fisher Scientific, Waltham, MA, USA) and the following primers: Gapdh-Fw 5'-GGAGAAACCTGCCAAGTATGA-3'; Gapdh-Rv 5' - T CCTCAGTGTAGCCCAAGA-3'; Gfi1-Fw 5' - AATGCA GCAAGGTGTTCTC-3'; Gfi1-Rv 5' - CTTACAGTCAAA GCTGCGT-3'.

\section{Immunostaining}

Progeny from a cross between $\mathrm{Gfi1}^{\mathrm{Cre} /+}$ mice and TdTomato reporter mice Ai14 were euthanized at P1 and their livers were harvested. Following fixation in $4 \%$ paraformaldehyde overnight at $4{ }^{\circ} \mathrm{C}$, livers were cryoprotected through incubation in PBS with increasing amount of sucrose before being embedded in O.C.T. compound (Scigen, Gardena, CA, USA). Ten $\mu \mathrm{m}$ cryosections were permeabilized with PBS supplemented with $0.2 \%$ Tween-20 for $1 \mathrm{~h}$ at room temperature and incubated with Alexa Fluor ${ }^{\mathrm{Tm}} 488$ Phalloidin (1/800, Thermo Fisher Scientific, Waltham, MA, USA) and DAPI $(1 / 20,000$, Thermo Fisher Scientific, Waltham, MA, USA). Samples were mounted with ProLong Gold antifade reagent (Thermo Fisher Scientific, Waltham, MA, USA). Images were acquired using a Nikon Eclipse E600 microscope (Nikon, Tokyo, Japan) equipped with a Lumenera Infinity 3 camera (Lumenera, Ottawa, ON).

\section{RNA-Seq library construction}

Below are the experimental methods for RNA-Seq library construction. We followed the manufacturer's instructions with minor modifications, as noted below. The shearing approach was not altered and remains different between kits.

\section{Ovation ${ }^{\circledR}$ RNA-Seq system V2 combined with TruSeq RNA library prep kit v2}

We performed a hybrid library preparation by using Ovation $^{\oplus}$ RNA-Seq System V2 (NuGEN, San Carlos, CA, USA) to synthesize cDNA and the TruSeq RNA Library Preparation Kit v2 to construct the sequencing library (Illumina, San Diego, CA, USA), consistent with the NuGEN manufacturer protocol (See Additional file 1: Table S1). Briefly, $4 \mathrm{ng}$ of total RNA or RiboTag IP RNA were used to synthesize cDNA following the NuGEN's instructions. Subsequently, $200 \mathrm{ng}$ of cDNA were sheared to an average size of $300 \mathrm{bp}$ with a Covaris E220
Focused-Ultrasonicator (Covaris Inc., Woburn, MA, USA). Following the manufacturer protocol, the library was prepared from the sheared cDNA using the Illumina TruSeq RNA Library Prep Kit with 8 cycles of PCR.

\section{SMARTer ${ }^{\circledast}$ stranded total RNA-Seq kit-Pico input mammalian}

Four nanograms of RNA were used as input material and libraries were prepared by following the SMARTer Stranded Total RNA-Seq kit-Pico Input Mammalian user manual (Takara Bio USA, Mountain View, CA, USA). In brief, samples were fragmented at $94{ }^{\circ} \mathrm{C}$ for $4 \mathrm{~min}$ prior to first-strand synthesis. Illumina adaptors and indexes were added to single-stranded cDNA via 5 cycles of PCR. Libraries were hybridized to R-probes for fragments originating from ribosomal RNA to be cleaved by ZapR. The resulting ribo-depleted library fragments were amplified with 15 cycles of PCR.

\section{SMART-Seq ${ }^{\circledR}$ v4 ultra ${ }^{\circledR}$ low input RNA kit for sequencing}

Two types of libraries were prepared by using $4 \mathrm{ng}$ or 250 pg RNA from each sample. Libraries were prepared by following the SMART-Seq v4 Ultra Low Input RNA Kit (Takara Bio USA, Mountain View, CA, USA) user manual. The cDNA was amplified with 11 cycles of PCR. Nextera XT kit (Nextera XT DNA Library Preparation Kit (Illumina, San Diego, CA) was used to make cDNA libraries suitable for Illumina sequencing.

\section{TruSeq RNA library prep kit v2}

Two types of libraries were prepared by using $70 \mathrm{ng}$ or 4 ng RNA from each sample. The 70 ng libraries were built using TruSeq RNA Library Prep Kit v2 (Illumina, San Diego, CA, USA) according to the manufacturer protocol. Size selection was performed using SPRIselect beads (Beckman Coulter, Indianapolis, IN, USA) and in-house calibration values (first round selection to select the upper or right limit of the distribution), salt unit equals to 0.427 and second round selection to select the lower or left limit of the distribution, salt unit = 0.455). The cDNA was amplified with 19 cycles of PCR. Libraries were prepared using 4 ng of RNA with modifications to the standard protocol by reducing the end-repair reaction to $1 / 2$ the recommended amounts of enzyme mix and sample volume. In addition A-tail ligation followed the standard protocol without the use of internal control mixes. Due to the low input amount, no size selection was applied to the $4 \mathrm{ng}$ libraries. The cDNA was amplified with 22 cycles of PCR. Libraries prepared using $70 \mathrm{ng}$ of RNA were prepared following the standard protocol and cDNA was amplified with 14 cycles as suggested by manufacture protocol. 

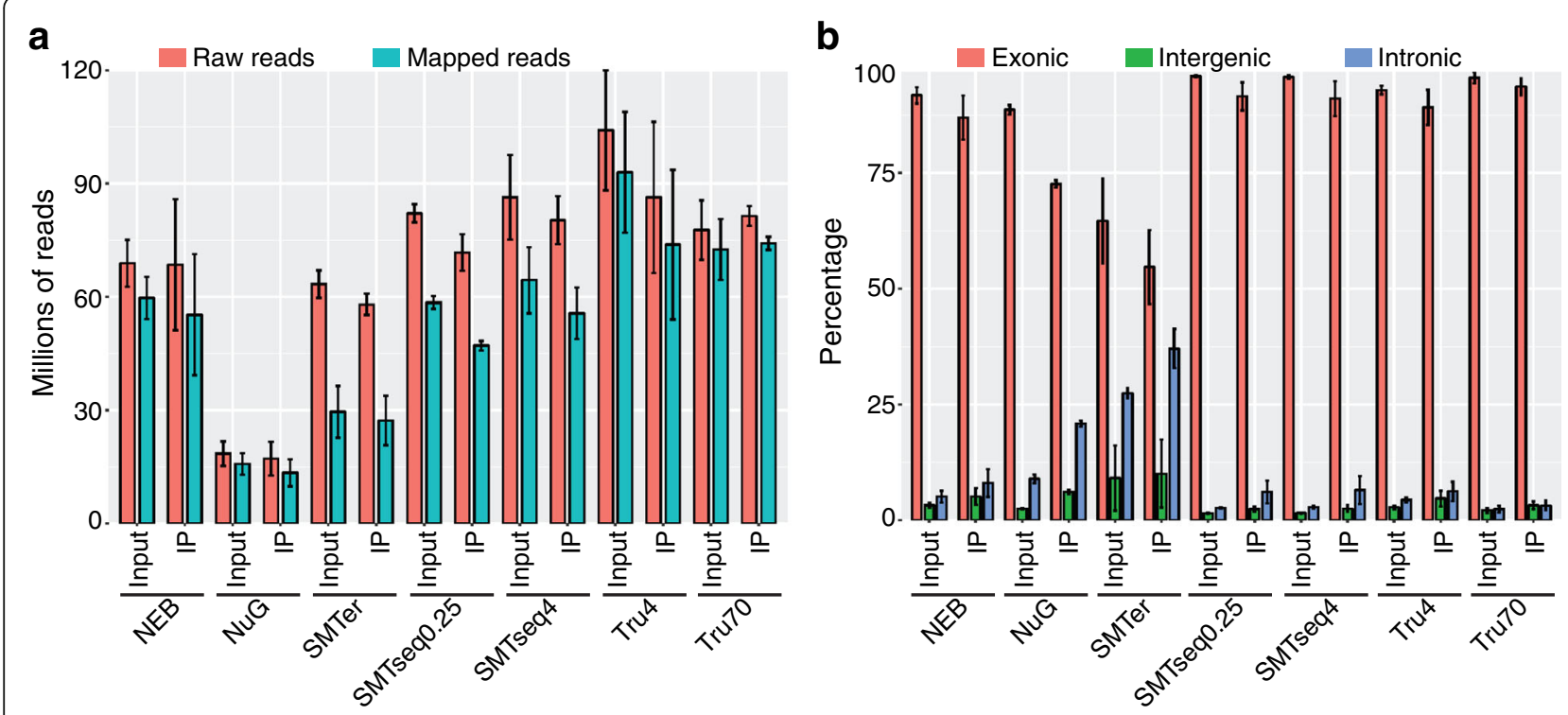

Fig. 1 Descriptive characteristics of raw and mapped reads. a Total number of raw reads and number of reads mapped to the mouse genome (mm10, GRCm38.84). b Percentage of reads mapped to exonic, intronic and intergenic regions. NEB: NEBNext ${ }^{\oplus}$ Ultra ${ }^{\mathrm{TM}}$, NuG: NuGEN Ovation ${ }^{\oplus}$, SMTer: SMARTer ${ }^{\oplus}$ Stranded; Tru4: TruSeq using 4 ng of RNA; Tru70: TruSeq using 70 ng of RNA. SMTseq4: SMART-Seq ${ }^{\oplus} 4$ using 4 ng of RNA; SMTseq0.25: SMART-Seq ${ }^{\circledR}$ v4 using 250 pg of RNA

\section{NEBNext ${ }^{\oplus}$ ultra $^{\mathrm{TM}}$ directional RNA library prep kit for Illumina}

Four nanograms of total RNA were used for NEBNext ${ }^{\circ}$ Ultra $^{\text {тм }}$ Directional RNA Library Prep Kit (New England Biolabs, Ipswich, MA, USA). Poly-A selection and cDNA synthesis were performed according to NEB protocol. The adaptors were diluted with a 1:30 ratio instead of the recommended 1:10 ratio. Size selection was performed using SPRIselect beads. (Beckman Coulter, Indianapolis, IN, USA) with in-house calibration values. The cDNA was amplified with 22 cycles of PCR.

\section{Sequencing}

Samples prepared by TruSeq, NEB, NuGEN and SMARTer were sequenced at the Institute for Genome Sciences (IGS) Genomics Resource Center (Baltimore, MD) on a HiSeq 4000 using 75 base read lengths in paired-end mode. Samples prepared by SMART-Seq v4 were sequenced by the Genomics and Computational Biology Core $(\mathrm{GCBC})$ at the National Institute on Deafness and Other Communication Disorders (NIDCD/NIH) on a HiSeq 1500 using a read length of 126 bases in paired-end mode.

\section{RNA-Seq analyses}

The Illumina adapters used during the library construction were removed from the reads using Trimmomatic [1]. In order to reduce the impact of lower quality reads on the alignment, all reads were trimmed to $60 \mathrm{bp}$
Table 1 Duplication rate of libraries prepared by different kits

\begin{tabular}{|c|c|c|c|c|c|c|}
\hline \multirow[t]{3}{*}{ Kit } & \multirow{3}{*}{$\begin{array}{l}\text { Sample } \\
\text { type }\end{array}$} & \multirow{3}{*}{$\begin{array}{l}\text { PCR cycles } \\
\text { for lib prep }\end{array}$} & \multicolumn{4}{|c|}{ Percent_Duplication (\%) } \\
\hline & & & \multicolumn{3}{|c|}{ replicate } & \multirow[t]{2}{*}{$\operatorname{avg}$} \\
\hline & & & 1 & 2 & 3 & \\
\hline \multirow[t]{2}{*}{$\mathrm{NEB}^{\mathrm{a}}$} & Input & 22 & 97 & 99 & 97 & 97 \\
\hline & $\mathbb{I P}$ & & 99 & 99 & 99 & 99 \\
\hline \multirow[t]{2}{*}{$\mathrm{NuG} / \mathrm{Tru}^{\mathrm{b}}$} & Input & 8 & 52 & 53 & 52 & 53 \\
\hline & $\mathrm{IP}$ & & 36 & 31 & 26 & 31 \\
\hline \multirow[t]{2}{*}{ SMTer ${ }^{\mathrm{c}}$} & Input & 15 & 83 & 86 & 85 & 85 \\
\hline & $\mathbb{I P}$ & & 86 & 83 & 68 & 79 \\
\hline \multirow[t]{2}{*}{ Tru4 ${ }^{d}$} & Input & 22 & 67 & 77 & 71 & 72 \\
\hline & IP & & 85 & 82 & 99 & 89 \\
\hline \multirow[t]{2}{*}{ Tru70 } & Input & 19 & 90 & 94 & 92 & 92 \\
\hline & IP & & 98 & 97 & 96 & 97 \\
\hline \multirow[t]{2}{*}{ SMTseq $4^{f}$} & Input & 11 & 40 & 38 & 40 & 40 \\
\hline & IP & & 60 & 49 & 48 & 52 \\
\hline \multirow[t]{2}{*}{ SMTseq $0.25^{f}$} & Input & 11 & 37 & 37 & 41 & 38 \\
\hline & $\mathbb{I P}$ & & 59 & 50 & 47 & 52 \\
\hline
\end{tabular}

${ }^{\mathrm{a}}$ NEBNext ${ }^{\oplus}$ Ultra $^{\mathrm{TM}}$ Directional RNA Library Prep Kit for Illumina

${ }^{\mathrm{b}}$ Nugen Ovation ${ }^{\circledR}$ RNAseq System v2

'SMARTer ${ }^{\circledast}$ Stranded Total RNA-Seq Kit-Pico Input Mammalian User

${ }^{\mathrm{d}}$ TruSeq $^{\circledast}$ RNA sample preparation $\mathrm{v} 2,4 \mathrm{ng}$

${ }^{\mathrm{e}}$ TruSeq ${ }^{\oplus}$ RNA sample preparation $\mathrm{v} 2,70 \mathrm{ng}$

fSMART-Seq v4 Ultra Low Input RNA Kit 
using the FASTX Toolkit $\mathrm{v}-0.0 .13$ [25] resulting in a Phred-Quality-Score greater than 30 . The reads generated for each RNA sample were analyzed and compared using an Ergatis-based RNA-Seq analysis pipeline [26] where sequencing reads were aligned and annotated to the UCSC mouse reference genome (mm10, GRCm38.84) from Ensembl (http://www.ensembl.org) using TopHat $\mathrm{v}-2.0 .8$ [27] (maximum number of mismatches = 2; segment length $=30$; maximum multi-hits per read $=$ 25 ; maximum intron length $=50,000$ ) and the number of reads that aligned to the predicted coding regions were determined using HTSeq [28]. Bedtools (v-2.7.1) [29] was used to count the reads mapping to exons according to Ensembl gene annotations (March 2016, Mus_musculus.GRCm38.84, with 47,729 genes annotated). Read counts per million mapped reads values (CPM) [28] or reads per kilobase of transcripts per million mapped reads (RPKM) [30] were calculated and used for downstream analyses. 5' -3' exonic coverage was calculated using CollectRnaSeqMetrics component of picard-tools ( $\mathrm{v}-1.60$, https://broadinstitute.github.io/picard/), and duplication rate was calculated using EstimateLibraryComplexity of Picard-tools.

\section{Statistical analysis}

All plots were generated using $\mathrm{R}$ ( $\mathrm{v}-3.2 .4)$, including the following $\mathrm{R}$ packages ggplot2, ComplexHeatmap for producing bar plots or heat maps, and limma to generate Venn diagrams. The difference among groups in boxplots was evaluated based on the overlapping of the notch region [31]. The notch is defined as median $\mathrm{m} \pm 1.58 \mathrm{IQR} / \sqrt{\mathrm{n}}$
[31]. The significance test is evaluated using a non-parametric Wilcoxon test with $p<0.05$.

\section{Accession number}

All of the processed gene expression data from this study have been submitted to the NCBI Gene Expression Omnibus (GEO) under accession number GSE104213.

\section{Results}

\section{Sample preparation for sequencing}

In order to evaluate the efficiency of different library preparation kits with low amounts of RNA obtained after ribosome immunoprecipitation, we crossed RiboTag mice with Gfi1-Cre mice to obtain progeny that expressed HA-tagged ribosomes in cells with Gfil expression. We obtained RNA from liver because it is a tissue that, at least during embryogenesis, expresses Gfil, thus allowing for early induced recombination in a subset of the liver cells for permanent expression of a reporter gene [32] (Additional file 2: Figure S1a). Additionally, the size of the liver would provide enough material to test five different kits with varying amounts of starting RNA from individually processed samples. Livers were processed for HA-tagged ribosome immunoprecipitation (IP) followed by RNA extraction as previously described [16]. Prior to sequencing, the efficacy of the IP was confirmed by comparing the level of Gfil transcripts in the input and IP samples using real time RT-PCR (Additional file 2: Figure S1b). The profiles generated by the five different commercial library preparition kits, from four different manufacturers, were compared in this study (See Additional file 1: Table S1). NEBNext ${ }^{\circ}$ Ultra $^{\mathrm{ax}}$

Table 2 Number of features with CPM $>0$

\begin{tabular}{|c|c|c|c|c|c|c|c|c|c|}
\hline \multirow[t]{3}{*}{ Kit } & \multirow{3}{*}{$\begin{array}{l}\text { Sample } \\
\text { type }\end{array}$} & \multicolumn{4}{|c|}{ Number of features with coverage (CPM >0) } & \multicolumn{4}{|c|}{ Number of reads of all expressed features $(\mathrm{CPM}>0)$} \\
\hline & & \multicolumn{3}{|c|}{ Replicate } & \multirow[t]{2}{*}{ avg } & \multicolumn{3}{|l|}{ Replicate } & \multirow[t]{2}{*}{ avg } \\
\hline & & 1 & 2 & 3 & & 1 & 2 & 3 & \\
\hline \multirow[t]{2}{*}{ NEB } & Input & 13,543 & 12,464 & 11,419 & 12,475 & $22,373,163$ & $26,292,017$ & $22,305,929$ & $23,657,036$ \\
\hline & $\mathbb{P}$ & 11,059 & 12,165 & 14,331 & 12,518 & $16,668,355$ & $27,323,849$ & $15,122,257$ & $19,704,820$ \\
\hline \multirow[t]{2}{*}{$\mathrm{NuG}$} & Input & 16,946 & 16,458 & 15,624 & 16,343 & $5,529,417$ & $7,615,866$ & $7,482,819$ & $6,876,034$ \\
\hline & $\mathbb{I P}$ & 17,715 & 17,841 & 18,198 & 17,918 & $3,223,153$ & $5,319,712$ & $5,141,329$ & $4,561,398$ \\
\hline \multirow[t]{2}{*}{ SMTer } & Input & 12,281 & 10,184 & 10,929 & 11,131 & 759,221 & 762,562 & 750,159 & 757,314 \\
\hline & $\mathbb{P}$ & 12,422 & 11,168 & 15,684 & 13,091 & 701,059 & 645,414 & 464,199 & 603,557 \\
\hline \multirow[t]{2}{*}{ Tru4 } & Input & 19,906 & 19,863 & 19,631 & 19,800 & $35,368,451$ & $45,466,334$ & $31,299,550$ & $37,378,112$ \\
\hline & $\mathbb{I P}$ & 17,467 & 18,899 & 21,161 & 19,176 & $36,785,680$ & $28,571,125$ & $18,031,264$ & $27,796,023$ \\
\hline \multirow[t]{2}{*}{ Tru70 } & Input & 15,957 & 15,577 & 16,082 & 15,872 & $32,103,296$ & $33,797,108$ & $25,797,283$ & $30,565,896$ \\
\hline & $\mathbb{I P}$ & 14,430 & 15,213 & 15,680 & 15,108 & $31,150,532$ & $29,799,544$ & $27,902,766$ & $29,617,614$ \\
\hline \multirow[t]{2}{*}{ SMTseq4 } & Input & 19,111 & 19,287 & 19,630 & 19,343 & $23,573,272$ & $24,615,731$ & $30,217,161$ & $26,135,388$ \\
\hline & $\mathbb{I P}$ & 19,835 & 20,551 & 20,488 & 20,291 & $14,407,184$ & $19,577,709$ & $17,262,557$ & $17,082,483$ \\
\hline \multirow[t]{2}{*}{ SMTseq0.25 } & Input & 16,834 & 16,742 & 16,117 & 16,564 & $23,815,261$ & $24,347,672$ & $22,951,893$ & $23,704,942$ \\
\hline & $\mathbb{P}$ & 16,827 & 16,870 & 16,527 & 16,741 & $13,971,566$ & $15,047,219$ & $14,691,099$ & $14,569,961$ \\
\hline
\end{tabular}


Directional RNA Library Prep Kit for Illumina (NEB) with $4 \mathrm{ng}$ of RNA,NuGEN Ovation ${ }^{\circ}$ RNA-Seq System V2 with 4 ng of RNA (NuGEN) with 4 ng of RNA, TaKaRa SMARTer ${ }^{\circ}$ Stranded Total RNA-Seq Kit-Pico Input Mammalian with $4 \mathrm{ng}$ of RNA (SMARTer), TaKaRa SMART-Seq ${ }^{\circ}$ v4 Ultra ${ }^{\circ}$ Low Input RNA Kit for Sequencing with $4 \mathrm{ng}$ and $0.25 \mathrm{ng}$ of RNA (SMARTseq4 and SMARTseq0.25) and Illumina TruSeq RNA Library Prep Kit v2 with 4 ng and 70 ng of RNA (TruSeq4 and TruSeq70). Of these kits only the SMARTer kit produced strand specific libraries and we therefore did not analyze the data for strandness.

\section{Comparison of mapping efficiency and duplication rate}

The number of reads varied widely among samples being prepared by different library preparation kits. Input RNA samples generated 14.7 to 122 million pair-end reads $(2 \times$ $60 \mathrm{bp}$ ) and IP RNA samples generated 12 to 108 million pair-end reads $(2 \times 60 \mathrm{bp})$. Overall, fewer raw/mapped reads were generated when using the NuGEN kit. Of the raw reads, 12.5 to 111 million reads mapped to mouse genome for input RNA samples while 9.2 to 94.6 million reads mapped for IP RNA samples (Fig. 1a).

In order to evaluate the expression profile composition and library complexity, we assessed the duplication rate of the read pairs (Table 1) as lower duplication rates usually indicate a higher complexity of the sample and better representation of RNA present in a sample [20]. In this study, duplication rate ranged from 26 to $99 \%$ (Table 1). However, the duplication we observed was not well correlated with the numbers of PCR cycles and was more dependent on the library prep kit. For instance, while NEB and TruSeq4 samples both had the highest number of PCR cycles (22), their duplication rates differed (Table 1). Indeed, NEB-input samples had the highest duplication rate of $99 \%$ with the overall largest number of reads duplicated more than 200 times while the TruSeq4 samples had a duplication rate of $72 \%$ with a substantially smaller number of reads with greater than 200 duplications (Table 1 and Additional file 3: Figure S2).

\section{Detection of exonic, intergenic and intronic regions}

Among the mapped reads, SMARTer samples showed the lowest alignment to exonic regions. The percentage of reads aligned to exonic regions was greater than $85 \%$ in samples prepared with NEB, TruSeq and SMARTseq library kits and less than $70 \%$ in samples prepared with the SMARTer and NuGEN kits (Fig. 1b). As expected, the overall percentage of reads aligning to intronic regions detected in input samples was less than 10\% for most samples, except for samples prepared by the SMARTer kit, where more than $20 \%$ of the reads align to intronic regions. IP samples had roughly twice as many reads aligning to intronic regions, or $10 \%$ more intronic reads compared with the corresponding input RNA samples, which may suggest that the IP captures some immature mRNAs. In particular, the percentage of intronic reads from the SMARTer samples increased from $22 \%$ for the input RNA to $41 \%$ for the IP RNA. The percentage of intronic reads for the NuGEN samples ranged from $8 \%$ for the input RNA and $22 \%$ for the IP RNA.

\section{Number of genes being detected as expressed}

Because of the differences in sequencing efficiency and library complexity, we examined the number of features detected in samples prepared with each library kit. After removing ambiguous reads or reads mapped to multiple

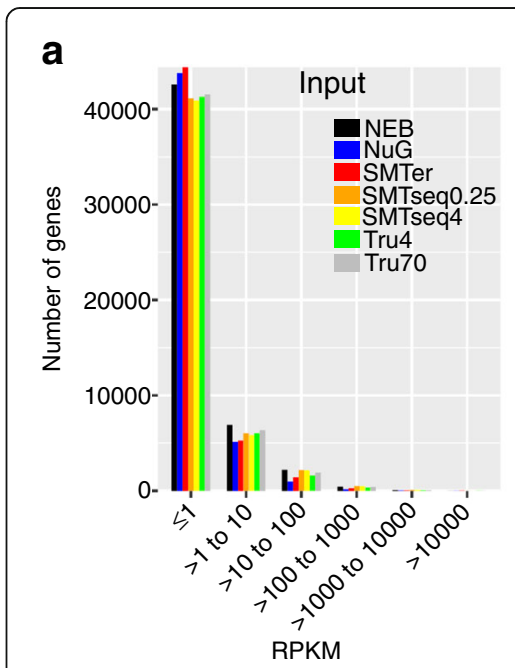

C
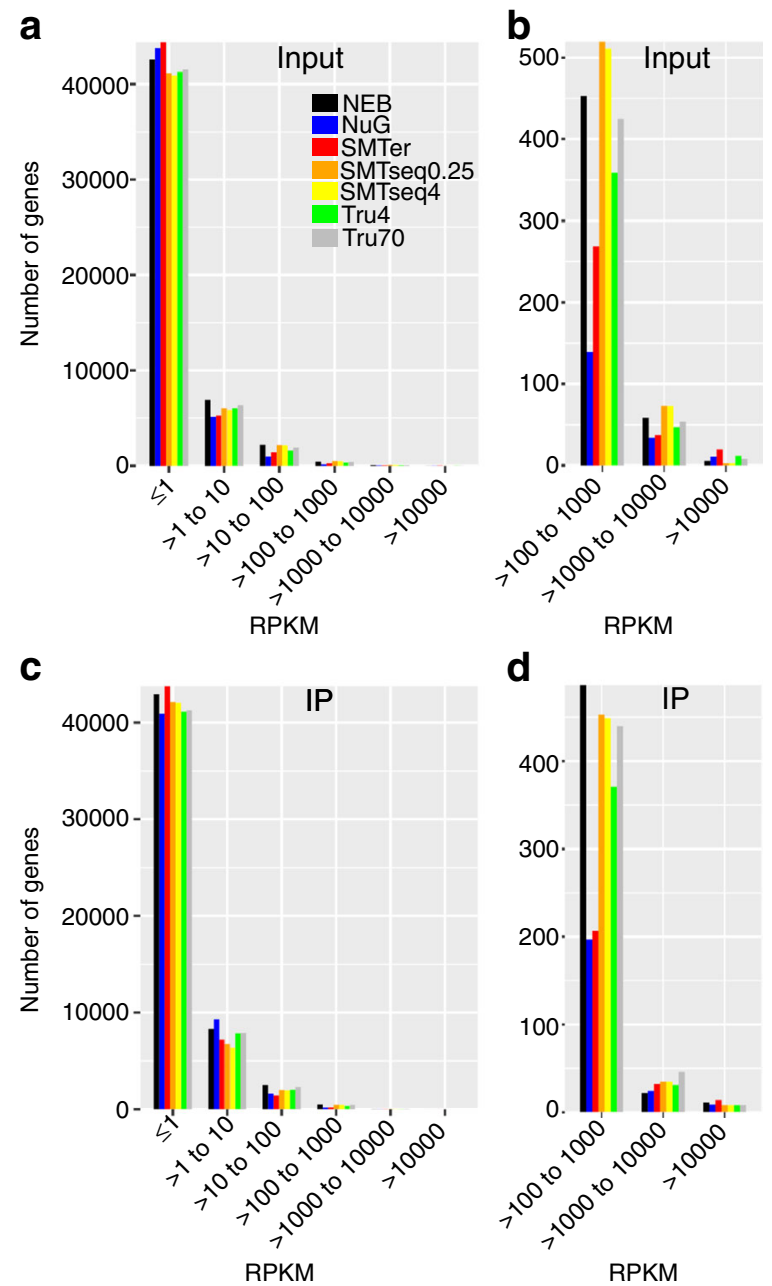

Fig. 2 Detected transcripts binned by RPKM. There were 33,399 transcripts detected in at least one input sample, 35,395 transcripts detected in at least one IP sample and all of these genes were used for the binning. Y-axis: number of transcripts detected in at least one replicate. $\mathbf{a}$ and $\mathbf{c}$ Number of genes binned by RPKM as detected by the different kits in the input and IP, respectively. $\mathbf{b}$ and $\mathbf{d}$ These plots are subsets of (a) and (c) focusing only on highly expressed transcripts (RPKM>100) 
features using HTSeq, we detected between 10,184 to 21,161 genes where the CPM values were greater than zero (Table 2). The corresponding average raw read counts ranged from 0.76 to 37 million reads. Fewer features were detected in SMARTer and NEB samples. All of the annotated genes $(47,729)$ were binned into 6 groups $(\mathrm{RPKM} \leq 1,1<\mathrm{RPKM} \leq 10,10<\mathrm{RPKM} \leq 100,100<$ $R P K M \leq 1000,1000<R P K M \leq 10,000$ and $R P K M>10,000$. Fig. 2). SMARTer, NuGEN, and NEB samples had more genes that were entirely missed or had low expression levels $(\mathrm{RPKM} \leq 1)$ in comparison to the other kits in the input samples (Fig. 2a). The SMARTer and NEB samples had more genes with a lower expression levels $(\mathrm{RPKM} \leq 1)$ also in the IP (Fig. 2c). The number of genes within RPKM range $(100-10,000)$ was relatively low in SMARTer and NuGEN samples (Fig. 2b, d). Conversely,
SMARTer samples contained more highly expressed genes (RPKM $>10,000)$ than others samples, but the majority of these were rRNA genes or genes encoding for hypothetical proteins (Fig. 2b, d and See Additional file 1: Table S2).

In order to compare the similarity of expression profiles of the different kits, we compared genes with at least 1 $\mathrm{CPM}$ in at least one replicate across all the samples. More than $60 \%$ of the genes were co-detected by all kits (Fig. 3). The median CPM for shared genes across all samples was 28 for input samples (Fig. 3a, c) and 36 for IP samples (Fig. 3b, d). Meanwhile, less than $10 \%$ of features were uniquely detected in NEB, NuGEN and TruSeq input samples, but over $20 \%$ of features were uniquely detected from the SMARTer samples. The median CPM of uniquely detected genes in SMARTer input samples was around 10, while the median for other kits was less than 3 .
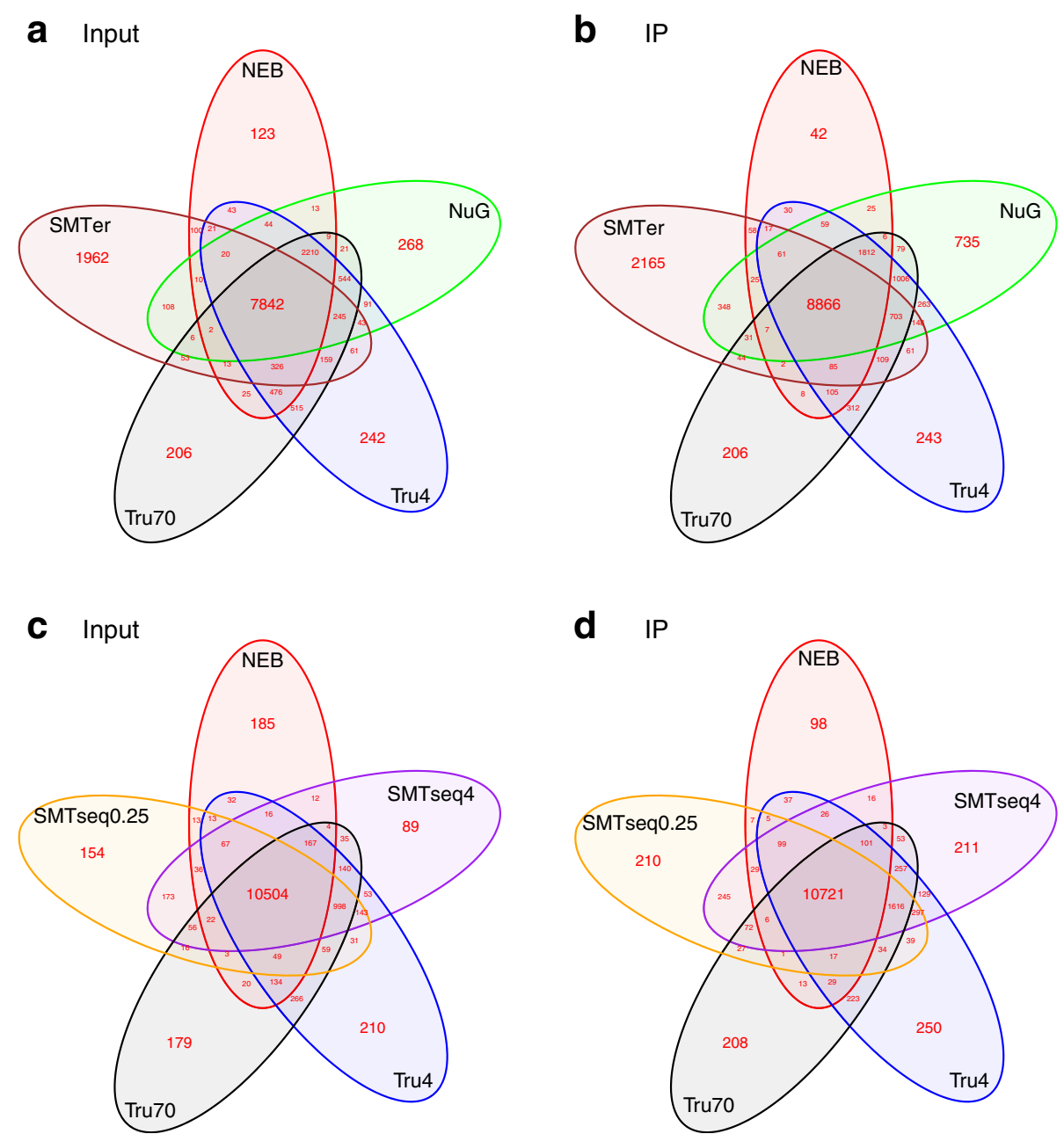

Fig. 3 Venn diagrams of identified features in the different libraries. The features with CPM $\geq 1$ in at least one out of 3 replicates were used to generate these plots. $\mathbf{a}$ and $\mathbf{c}$ represent input samples and $\mathbf{b}$ and $\mathbf{d}$ represent IP samples. Most transcripts were detected by all kits tested. However, a higher rate of agreement is seen between the NEB, TruSeq and SMART-Seq prepared samples. NEB: NEBNext $t^{\oplus}$ Ultra ${ }^{\mathrm{TM}}{ }^{,}$NuG: NuGEN Ovation ${ }^{\circledast}$, SMTer: SMARTer ${ }^{\circledR}$ Stranded; Tru4: TruSeq using 4 ng of RNA; Tru70: TruSeq using 70 ng of RNA. SMTseq4: SMART-Seq ${ }^{\circledR}$ V4 using 4 ng of RNA; SMTseq0.25: SMART-Seq ${ }^{\circledR}$ v using 250 pg of RNA 
Table 3 Descriptive statistic of non-coding detected features (CPM > =1)

\begin{tabular}{|c|c|c|c|c|c|c|c|c|c|c|c|c|c|}
\hline \multirow[t]{2}{*}{ Kit } & \multirow[t]{2}{*}{$\begin{array}{l}\text { Sample } \\
\text { type }\end{array}$} & \multicolumn{3}{|c|}{$\begin{array}{l}\text { Total features of ribosomal } \\
\text { RNA } \\
\text { Replicate }\end{array}$} & \multicolumn{3}{|c|}{$\begin{array}{l}\text { Average CPM of ribosomal } \\
\text { RNA } \\
\text { Replicate }\end{array}$} & \multicolumn{3}{|c|}{$\begin{array}{l}\text { Total features of ribosomal } \\
\text { RNA, lincRNA, microRNA } \\
\text { Replicate }\end{array}$} & \multicolumn{3}{|c|}{$\begin{array}{l}\text { Average CPM of ribosomal RNA, } \\
\text { lincRNA, microRNA } \\
\text { Replicate }\end{array}$} \\
\hline & & 1 & 2 & 3 & 1 & 2 & 3 & 1 & 2 & 3 & 1 & 2 & 3 \\
\hline \multirow[t]{2}{*}{$\overline{N E B}$} & Input & 3 & 3 & 3 & 73.87 & 67.33 & 72.15 & 233 & 186 & 140 & 46.01 & 29.22 & 28.34 \\
\hline & $\mathbb{I P}$ & 3 & 3 & 4 & 14.24 & 6.15 & 7.80 & 128 & 157 & 268 & 78.05 & 28.33 & 34.25 \\
\hline \multirow[t]{2}{*}{$\mathrm{NuG}$} & Input & 8 & 11 & 10 & 5533.70 & 5571.81 & 5603.65 & 552 & 488 & 415 & 272.73 & 274.17 & 276.54 \\
\hline & $\mathbb{I P}$ & 13 & 11 & 17 & 1803.34 & 820.73 & 863.91 & 583 & 593 & 637 & 97.91 & 48.26 & 51.37 \\
\hline \multirow[t]{2}{*}{ SMTer } & Input & 8 & 5 & 6 & 4282.62 & 7858.83 & 4641.15 & 440 & 359 & 377 & 247.75 & 429.65 & 261.04 \\
\hline & $\mathbb{I P}$ & 12 & 6 & 17 & 4880.27 & 7906.14 & 3936.78 & 467 & 411 & 599 & 282.51 & 429.64 & 240.08 \\
\hline \multirow[t]{2}{*}{ Tru4 } & Input & 17 & 17 & 13 & 232.50 & 120.94 & 169.47 & 804 & 786 & 767 & 13.96 & 7.59 & 10.29 \\
\hline & $\mathbb{I P}$ & 8 & 7 & 19 & 415.93 & 129.19 & 168.59 & 546 & 650 & 927 & 26.49 & 8.94 & 11.45 \\
\hline \multirow[t]{2}{*}{ Tru70 } & Input & 6 & 4 & 4 & 102.39 & 28.55 & 58.50 & 410 & 363 & 406 & 6.34 & 2.08 & 3.81 \\
\hline & IP & 3 & 3 & 4 & 141.53 & 54.91 & 65.21 & 265 & 295 & 341 & 9.24 & 3.97 & 5.10 \\
\hline \multirow[t]{2}{*}{ SMTseq4 } & Input & 13 & 13 & 21 & 214.51 & 159.76 & 185.17 & 750 & 764 & 803 & 11.78 & 8.93 & 10.54 \\
\hline & $\mathbb{I P}$ & 7 & 11 & 10 & 851.62 & 63.75 & 92.92 & 844 & 881 & 889 & 46.39 & 5.91 & 8.73 \\
\hline \multirow[t]{2}{*}{ SMTseq0.25 } & Input & 6 & 7 & 3 & 224.50 & 191.46 & 235.20 & 531 & 515 & 461 & 12.32 & 10.52 & 13.04 \\
\hline & $\mathbb{P}$ & 6 & 6 & 7 & 886.27 & 73.72 & 99.28 & 476 & 502 & 457 & 48.03 & 6.43 & 8.88 \\
\hline
\end{tabular}

A similar trend is observed in IP samples (Fig. 3b, $\mathrm{d}$ and See Additional file 1: Table S3).

We also grouped all of the detected features into ribosomal RNA, non-coding (ribosomal RNA, lincRNA, microRNA) and protein-coding groups. The average CPM of ribosomal RNA and non-coding gene groups were 2-fold higher in NuGEN and SMARTer samples than in other samples (Table 3). Conversely, the average CPMs for the protein-coding group were similar across most samples, except for SMARTer prepared samples
(Table 4). By comparing IP samples with input samples, it is interesting that the CPMs of IP samples are relatively lower than input samples, except for NuGEN prepared samples.

\section{Coverage uniformity relative to $5^{\prime}$ and $3^{\prime}$ ends}

The evenness of transcript coverage was calculated by dividing the mean coverage of first (last) 100 bases $\left(5^{\prime}\right.$ or $3^{\prime}$ ) of transcripts divided by the mean coverage of all bases across the corresponding transcript (Fig. 4). The median

Table 4 Descriptive statistic of protein-coding detected features (CPM $\geq 1$ )

\begin{tabular}{|c|c|c|c|c|c|c|c|}
\hline \multirow[t]{2}{*}{ Kit } & \multirow[t]{2}{*}{$\begin{array}{l}\text { Sample } \\
\text { type }\end{array}$} & \multicolumn{3}{|c|}{$\begin{array}{l}\text { Total features of protein-coding RNA } \\
\text { Replicate }\end{array}$} & \multicolumn{3}{|c|}{$\begin{array}{l}\text { Average CPM of protein-coding RNA } \\
\text { Replicate }\end{array}$} \\
\hline & & 1 & 2 & 3 & 1 & 2 & 3 \\
\hline \multirow[t]{2}{*}{ NEB } & Input & 11,316 & 10,551 & 9789 & 36.51 & 37.77 & 37.83 \\
\hline & $\mathbb{P}$ & 8740 & 10,252 & 12,435 & 34.13 & 37.84 & 37.39 \\
\hline \multirow[t]{2}{*}{$\mathrm{NuG}$} & Input & 10,921 & 10,828 & 10,656 & 19.61 & 19.50 & 19.32 \\
\hline & $\mathrm{IP}$ & 12,970 & 13,142 & 13,318 & 32.65 & 36.35 & 36.12 \\
\hline \multirow[t]{2}{*}{ SMTer } & Input & 11,136 & 9368 & 10,019 & 21.47 & 7.90 & 20.48 \\
\hline & IP & 11,826 & 11,860 & 11,824 & 18.88 & 7.90 & 22.02 \\
\hline \multirow[t]{2}{*}{ Tru4 } & Input & 11,826 & 11,860 & 11,824 & 38.90 & 39.38 & 39.18 \\
\hline & IP & 12,360 & 12,842 & 12,941 & 37.97 & 39.28 & 39.09 \\
\hline \multirow[t]{2}{*}{ Tru70 } & Input & 11,690 & 11,691 & 11,611 & 39.47 & 39.79 & 39.66 \\
\hline & IP & 11,996 & 12,493 & 12,660 & 39.26 & 39.65 & 39.56 \\
\hline \multirow[t]{2}{*}{ SMTseq4 } & Input & 11,659 & 11,499 & 11,578 & 39.07 & 39.28 & 39.16 \\
\hline & $\mathbb{P}$ & 12,443 & 12,579 & 12,587 & 36.50 & 39.51 & 39.01 \\
\hline \multirow[t]{2}{*}{ SMTseq0.25 } & Input & 11,500 & 11,475 & 11,384 & 39.03 & 39.16 & 38.97 \\
\hline & $\mathbb{I P}$ & 12,648 & 12,846 & 12,940 & 36.38 & 39.47 & 39.29 \\
\hline
\end{tabular}




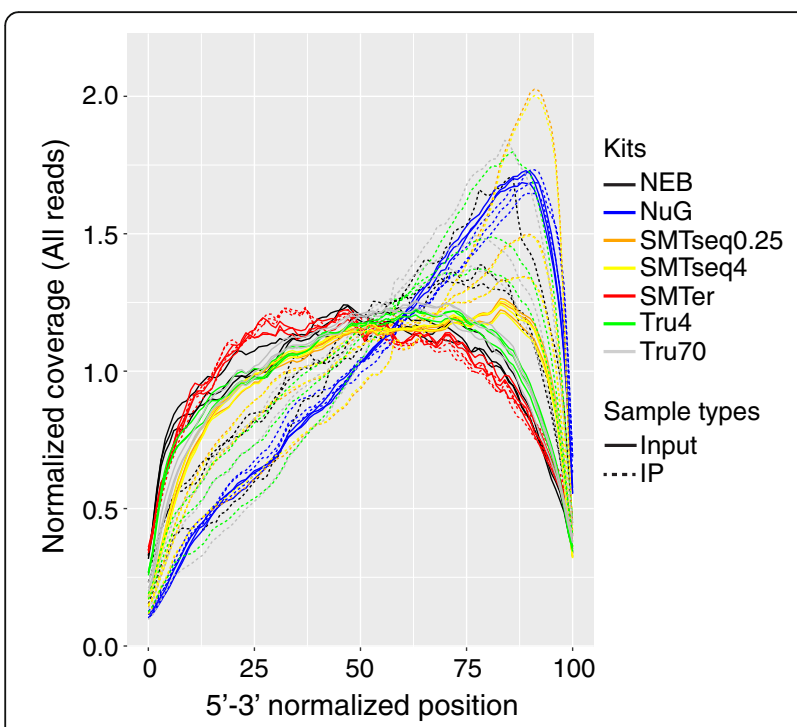

Fig. 4 Distribution of normalized mean expression of the first (last) 100 bases of transcripts (in $5^{\prime}->3^{\prime}$-orientation). $X$ axis represents the 5'-3' normalized position; $Y$ axis represents normalized coverage. NEB: NEBNext ${ }^{\oplus}$ Ultra ${ }^{\text {TM }}$, NuG: NuGEN Ovation ${ }^{\oplus}$, SMTer: SMARTer ${ }^{\oplus}$ Stranded; Tru4: TruSeq using 4 ng of RNA; Tru70: TruSeq using 70 ng of RNA. SMTseq4: SMART-Seq ${ }^{\oplus}$ v using 4 ng of RNA; SMTseq0.25: SMART-Seq ${ }^{\oplus}$ v using $250 \mathrm{pg}$ of RNA. Yellow and orange: SMTseq samples; Red: SMTer samples; Black: NEB samples; Blue: NuG samples; Green and grey: TruSeq samples. Solid: Input samples. Dotted: Ribo-IP samples

was calculated and plotted for the 1000 most highly expressed transcripts. Most of the input RNA samples showed even coverage from $5^{\prime}$ to $3^{\prime}$ end, except for all NuGEN samples which had pronounced increase in coverage at the $3^{\prime}$ end. Additionally, consistently higher coverage at the $3^{\prime}$ end was observed among IP RNA samples, except for SMARTer samples with even coverage across $5^{\prime}$ and $3^{\prime}$ extremities.

\section{Similarity of expression profiles}

In order to assess the similarity of expression profiles being generated by different library preparation kits, we applied Spearman correlation coefficients as a measure of similarity. The Spearman coefficient was calculated based on the rank of the CPM value as opposed to using the absolute values. This was done to accommodate the difference in CPM values due to differences in duplication rates observed among the kits (Fig. 5). The correlation coefficient for input samples ranged from 0.5 to 0.9 , where SMARTseq profiles were better correlated with TruSeq70 than others (Spearman correlation coefficient $\geq$ $0.9)$. SMARTer samples had the lowest correlation (0.5) with the control library TruSeq70 (Fig. 5 and See Additional file 1: Table S4). Overall, as expected, input profiles are less correlated to corresponding IP profiles (See Additional file 1: Table S4). When we compared input samples with corresponding IP samples for each individual kit respectively, all input samples were clustered separately from IP samples except for the SMARTer samples (Additional file 4: Figure S3).

Although two different amounts of RNA were used for the TruSeq library kit, TruSeq 4 ng samples were well correlated with TruSeq $70 \mathrm{ng}$ samples (Spearman correlation coefficient was $0.96 \pm 0.002$ for input; $0.946 \pm 0.01$ for IP). Similarly, the SMARTseq samples with $0.25 \mathrm{ng}$ and $4 \mathrm{ng}$ were highly correlated (Spearman correlation coefficient was $0.95 \pm 0.004$ for input and $0.95 \pm 0.005$ for IP) (See Additional file 1: Table S4).

\section{Transcript enrichment is better represented than transcript depletion in the IP samples}

We evaluated the robustness of different kits for detecting enrichment (IP/input RNA $>1$ ) or depletion (IP/input RNA $<1$ ) of transcripts in the translatome (IP samples) compared to the transcriptome (input samples). Features with raw read counts $\geq 20$ in input samples and with an enrichment or depletion factor $\geq 2$ were included as enriched (IP/Input $\geq 2$ ) or depleted transcripts (IP/Input $\leq 0.5$ ). Of note, more transcripts were enriched than depleted (Fig. 6a and Additional file 5: Figure S4). NuGEN produced the greatest number of enriched transcripts (mean 4270) and smallest number of depleted transcripts (mean 74) as compared with other kits (Fig. 6b). Among the enriched transcripts from NuGEN, $60 \%$ were enriched less than 4-fold whereas only $25 \%$ of transcripts prepared by other kits were enriched less than 4-fold (Fig. 6b). NEB samples had the highest percentage of enriched/depleted transcripts $(\log 2($ IP/INPUT $)>5$ or $\log 2($ INPUT/IP $)>5$ ) when compared to samples obtained from the other kits (Fig. 6b, Fig. 7a). Conversely, the enrichment profile of the SMARTer samples showed fewer enriched or depleted transcripts compared with the rest of the samples. Indeed, when plotting for the top 50 enriched transcripts (Fig. 7b), the median enrichment value for the SMARTer profile was significantly lower than other profiles $(p<0.05)$.

We also compared the number of transcripts being enriched or depleted across samples (Additional file 6: Figure S5). NuGEN had the highest number of uniquely enriched transcripts that were detected (accounting for $25 \%$ of its total enriched transcripts, $95 \%$ of which are protein-coding genes). TruSeq4 and TruSeq70 had around 5\% uniquely enriched transcripts (Additional file 6: Figure S5a,b).

We also clustered all the transcripts based on the rank of enrichment factor or depletion factor greater than 2 in at least one sample (Fig. 8). As expected, the profiles for TruSeq4 and TruSeq70 were most similar to each other (Spearman correlation coefficient $>0.7$ ). The same is true for SMARTseq4 and SMARTseq0.25. On the 


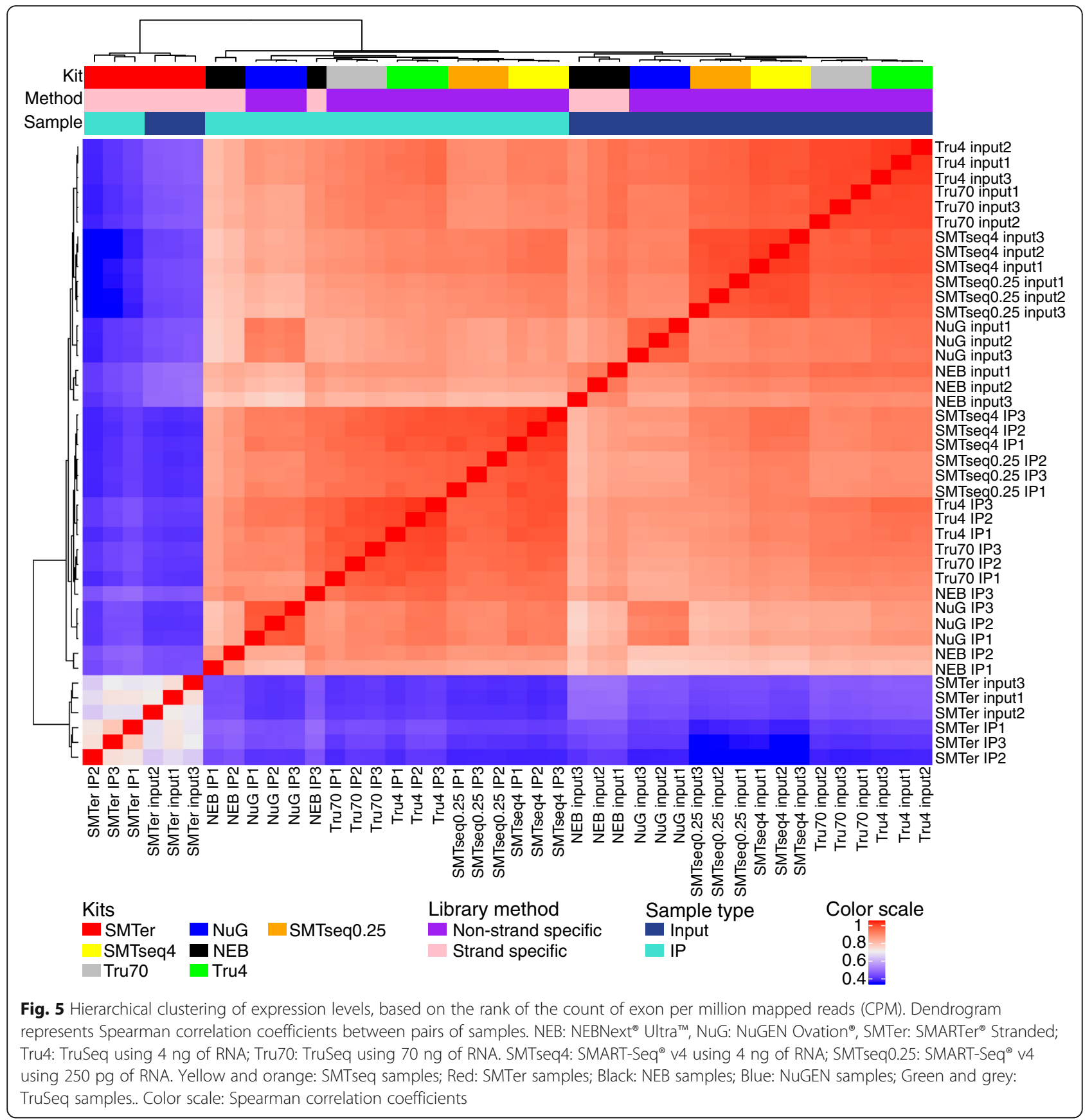

other hand, the enrichment/depletion profile for SMARTer was the least similar to the other profiles (Spearman correlation coeffienct $<0.2$ ).

\section{Transcript-length dependent enrichment/depletion}

We examined whether the enrichment or depletion effects observed in the translatome were affected by the transcript length. Based on size distribution of the enriched/ depleted transcripts (the majority being between 0.5 and $10 \mathrm{~kb}$, Additional file 7: Figure S6), we grouped the transcripts into four bins $(\leq 0.5 \mathrm{~kb}, 0.5-1 \mathrm{~kb}, 1 \mathrm{~kb}-10 \mathrm{~kb}$, and >
$10 \mathrm{~kb}$ ) (Fig. 9). The median enrichment for transcripts was relatively higher in the longer transcript $(>10 \mathrm{~kb})$ except in TruSeq70 samples (Fig. 9a). Within each transcript length bin, the median enrichment effect from NuGEN and SMARTer samples was much higher than TruSeq70 samples for transcripts less than $10 \mathrm{~kb}$ (Fig. 9b). For longer transcripts $(>10 \mathrm{~kb}), \mathrm{NEB}, \mathrm{NuGEN}$, SMARTer and SMARTseq samples had a median enrichment that is much higher than those of TruSeq70 (Fig. 9b). Additionally, the enrichment effect for NEB samples distributed wider than all the other samples (Fig. 9). 

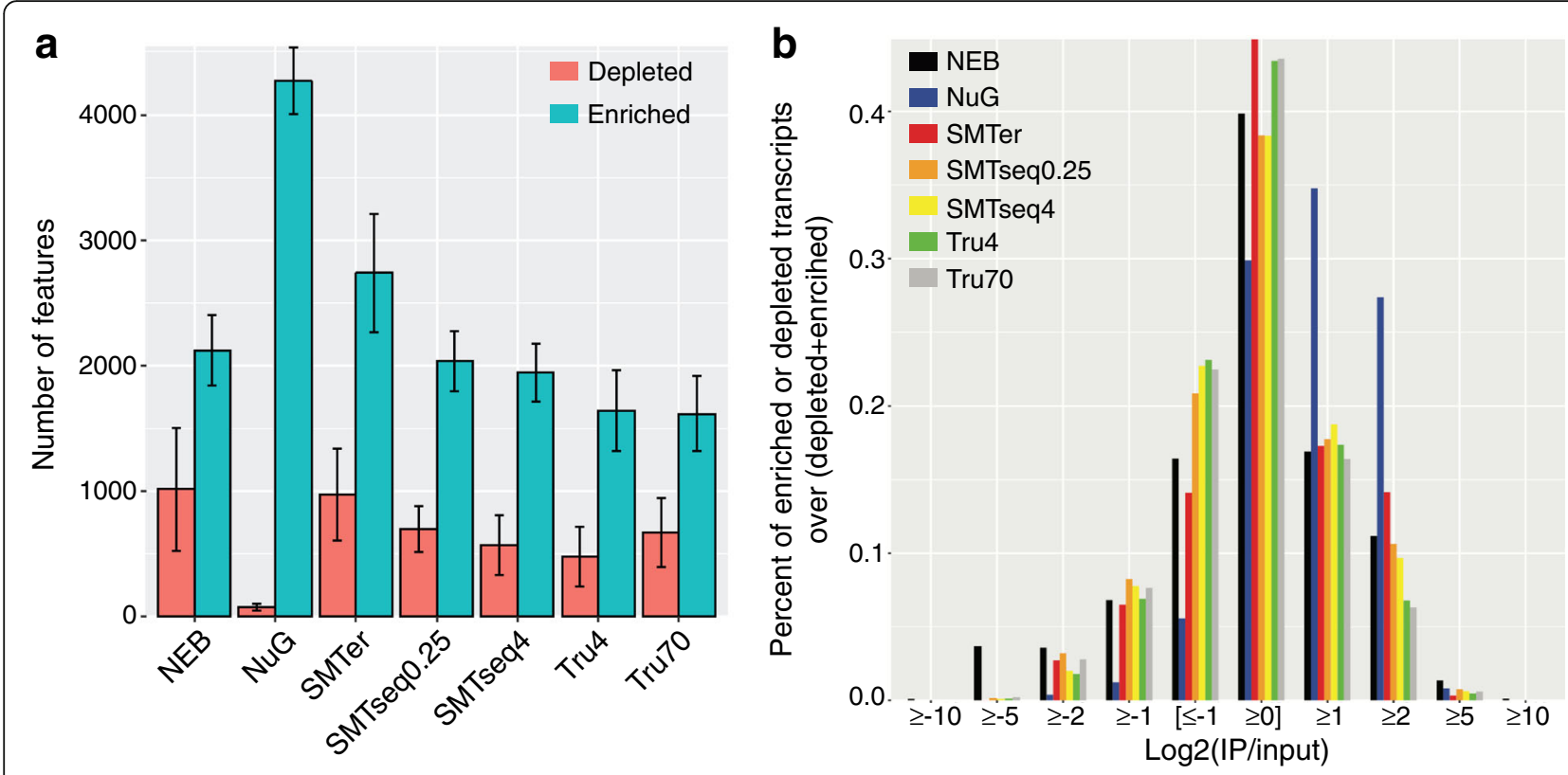

Fig. 6 Descriptive characteristic of enrichment or depletion profiles as generated by the different library preparation kits. Genes which have at least 20 raw reads in the input samples and a ratio of IP/Input $\geq 2$ or Input/IP $\geq 2$ were used to generate the plots. a Total number of transcripts enriched or depleted. $\mathbf{b}$ Percentage of enriched or depleted transcripts grouped into different bins. X-axis: log2(IP/input), Y-axis: percentage of genes in each bin over whole population. NEB: NEBNext ${ }^{\oplus}$ Ultra $^{\text {TMM }}$, NuG: NuGEN Ovation ${ }^{\oplus}$, SMTer: SMARTer ${ }^{\circledast}$ Stranded; Tru4: TruSeq using 4 ng of RNA; Tru70: TruSeq using 70 ng of RNA. SMTseq4: SMART-Seq ${ }^{\oplus}$ v using 4 ng of RNA; SMTseq0.25: SMART-Seq ${ }^{\circledR} 4$ using 250 pg of RNA. Yellow and orange: SMTseq samples; Red: SMTer samples; Black: NEB samples; Blue: NuGEN samples; Green and grey: TruSeq samples

A similar trend was also observed in depleted transcripts. Across all transcript lengths, the range of depletion effect for NuGEN and SMARTer samples was less than for other samples (Fig. 10a). For NEB samples, the depletion effect distribution was wider than all the other samples (Fig. 10b). For longer transcripts (>10 kb), NEB, NuGEN, SMARTer and SMARTseq samples showed fewer depletion effects than those from TruSeq70 (Fig. 10b).

\section{Discussion}

In this study, we compared five library-preparation kits for RNA-seq, using low-quantity input RNA or RiboTag IP RNA, by applying a comprehensive set of quality
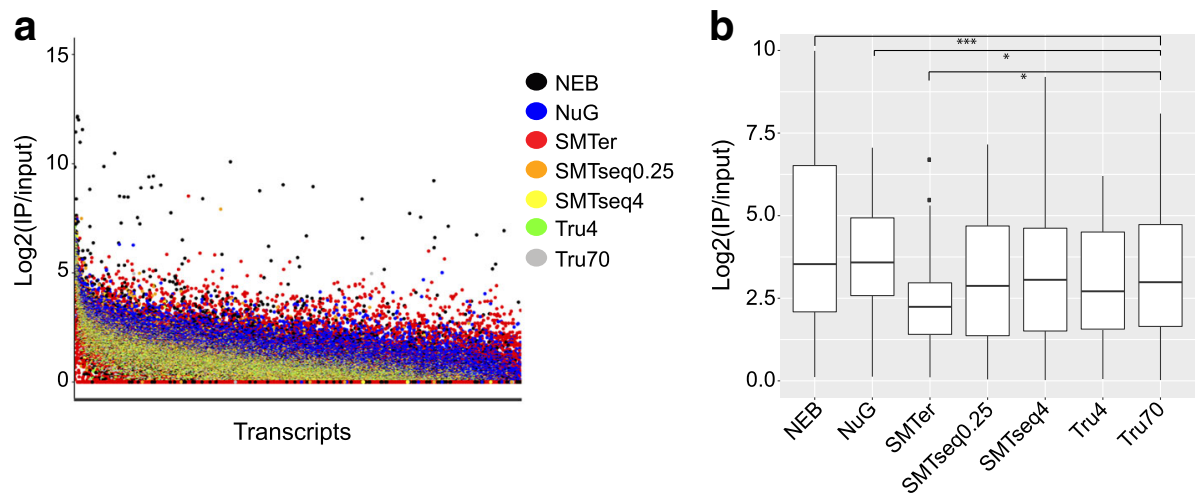

Fig. 7 Enrichment profiles and top 50 enriched transcripts. a Enrichment factor of transcripts are sorted in decreasing order based on log2 (IP) input). X-axis:transcripts, Y-axis:log2 value of enrichment (IP/Input). b Boxplot of top 50 enriched transcripts. NEB: NEBNext ${ }^{\oplus}$ Ultra ${ }^{\mathrm{TM}}$, NuG: NuGEN Ovation ${ }^{\oplus}$ SMTer: SMARTer ${ }^{\oplus}$ Stranded; Tru4: TruSeq using 4 ng of RNA; Tru70: TruSeq using 70 ng of RNA. SMTseq4: SMART-Seq ${ }^{\circledR} 4$ using 4 ng of RNA; SMTseq0.25: SMART-Seq ${ }^{\oplus}$ v4 using 250 pg of RNA. Yellow and orange: SMTseq samples; Red: SMTer samples; Black: NEB samples; Blue: NuGEN samples; Green and grey: TruSeq samples 


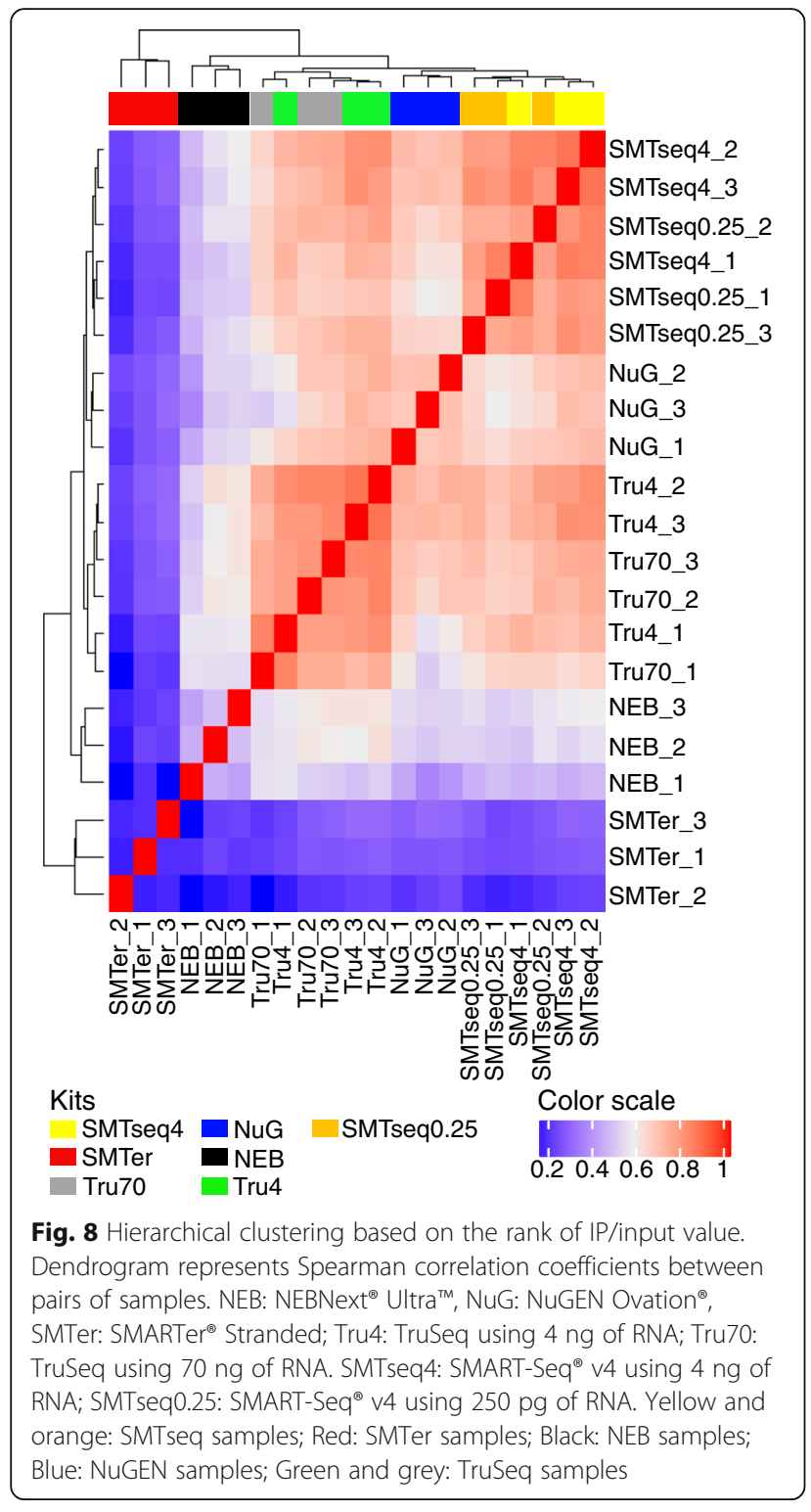

measures. One of the major differences among library preparation kits was whether oligo (dT) is used to select mRNA. Among the kits tested, the NEBNext ${ }^{\circ}$ Ultra $^{\mathrm{m}}{ }^{\circ}$, the Illumina TruSeq ${ }^{\circ}$ and the TaKaRa SMART-Seq ${ }^{\circledR}$ v4 Ultra ${ }^{\circ}$ use oligo-dT primers to select for polyA mRNA. Conversely, the TaKaRa SMARTer ${ }^{\circ}$ kit depends on locked nucleic acid (LNA) technology and random primers to capture both products with classical long polyA $(+)$ and those with short poly(A) tails or polyA(-) transcripts and employs a ribosomal depletion step. Although the NuGEN Ovation ${ }^{\circ}$ V2 kit uses a combination of semi-random hexamers and a poly-dT chimeric primer for 1st strand cDNA amplification in an effort to mitigate bias, 3' end bias was still observed. Shanker, et al.(2015), also observed 3' end bias using the NuGEN Ovation V2 kit with low input samples [22]. Interesingly, we observed greater 3' end bias in IP samples (except for SMARTer prepared samples) than in the input samples, possibly suggesting some degradation of the RNA.

A higher percentage of reads mapped to the intronic and intergenic region in the samples derived from the TaKaRa SMARTer ${ }^{\circ}$ kit in comparison to samples derived from the other five kits. Adiconis, et al., 2013 [23], also found a similar difference by comparing the SMARTer ${ }^{\circ}$ kit to the TruSeq ${ }^{\circ}$ kit. In our study, among the top- 100 highly expressed transcripts in SMARTer samples, 30\% were miRNA, lincRNA and rRNA. It is known that the source of miRNA or lincRNA is mainly from intergenic or intronic region, and that certain ribosomal RNAs generated by RNA polymerase I and III are without polyA tails. Therefore, we propose that the SMARTer kit may be useful for studies which aim to focus on poly(A) negative transcripts or transcripts derived from non-exon-coding regions.

We observed lower duplication rates for the SMART-Seq ${ }^{\circ}$ 4 Ultra (SMARTseq) and NuGEN prepared samples as compared to samples prepared with the other kits. A possible explanation to this observed lower duplication rate may relate to the protocols of these two kits. The mRNA is pre-amplifed to cDNA, before fragmentation, making the duplication rate resulting from the amplification harder to identify based on mapping position. Conversely, in other methods including the TruSeq kit, mRNA is fragmented first and the amplification only happens during the library construction step, making it easier to identify duplication based on the mapping position. For this study, the comparisons among different kits were achieved by using the rank-based method without removal of duplicate reads. Parekh et al., also showed that removal of duplicates improved neither accuracy nor precision and can actually worsen the power and the False Discovery Rate (FDR) for differential gene expression [33].

While Combs et al., (2015) [34] reported the use of the TruSeq kit with $100 \mathrm{ng}$ of RNA, our modification of the TruSeq protocol provides the possibility to use the kit with RNA amounts as low as 4 ng. Indeed, our study, for the first time, shows that with protocol modifications, TruSeq with 4 ng of RNA performs similarly to the TruSeq with $70 \mathrm{ng}$ of RNA with respect to the number of genes being captured and overall profile composition.

Comparing translatome (IP samples) against corresponding transcriptome (input samples), we find a relative higher intronic percentage in the translatome profiles, which might indicate that some non-mature RNA are precipitated during the IP process. Overall, we detected more enriched transcripts than depleted transcripts in the IP samples. Roh et al., report a similar result although the fold-change was greater in the depleted genes than the enriched genes [35]. This difference may result from the 

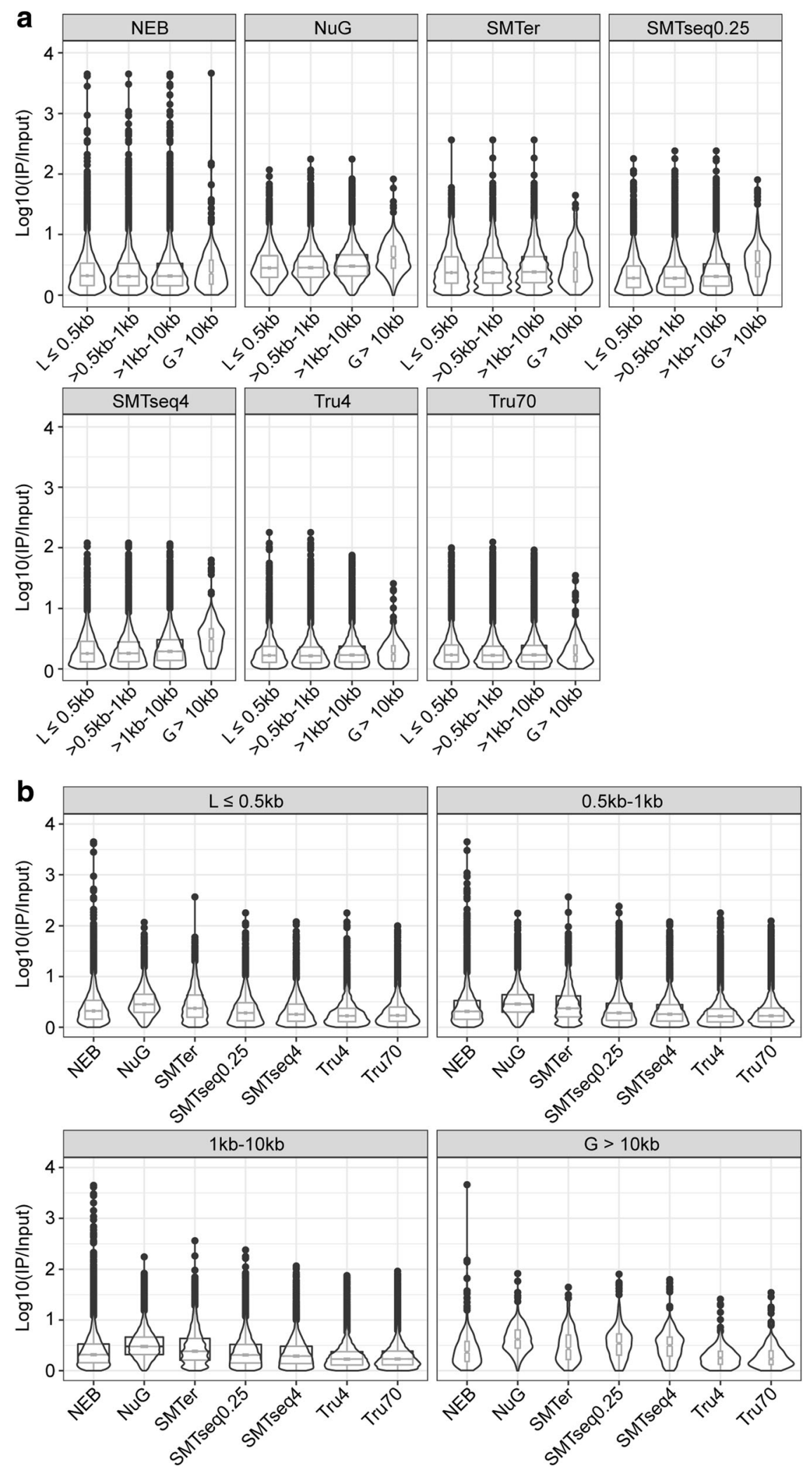

Fig. 9 Boxplot and violin plots of enriched features over different transcript lengths. a Boxplots of enrichment factor of different transcripts within each kit. $\mathbf{b}$ Boxplots of enrichment factor of different kits at each transcript length bin. The notch represents the median $\pm 1.58 \mathrm{IQR} / \sqrt{\mathrm{n}}$. The width of boxplot is proportion to sample size of each group 

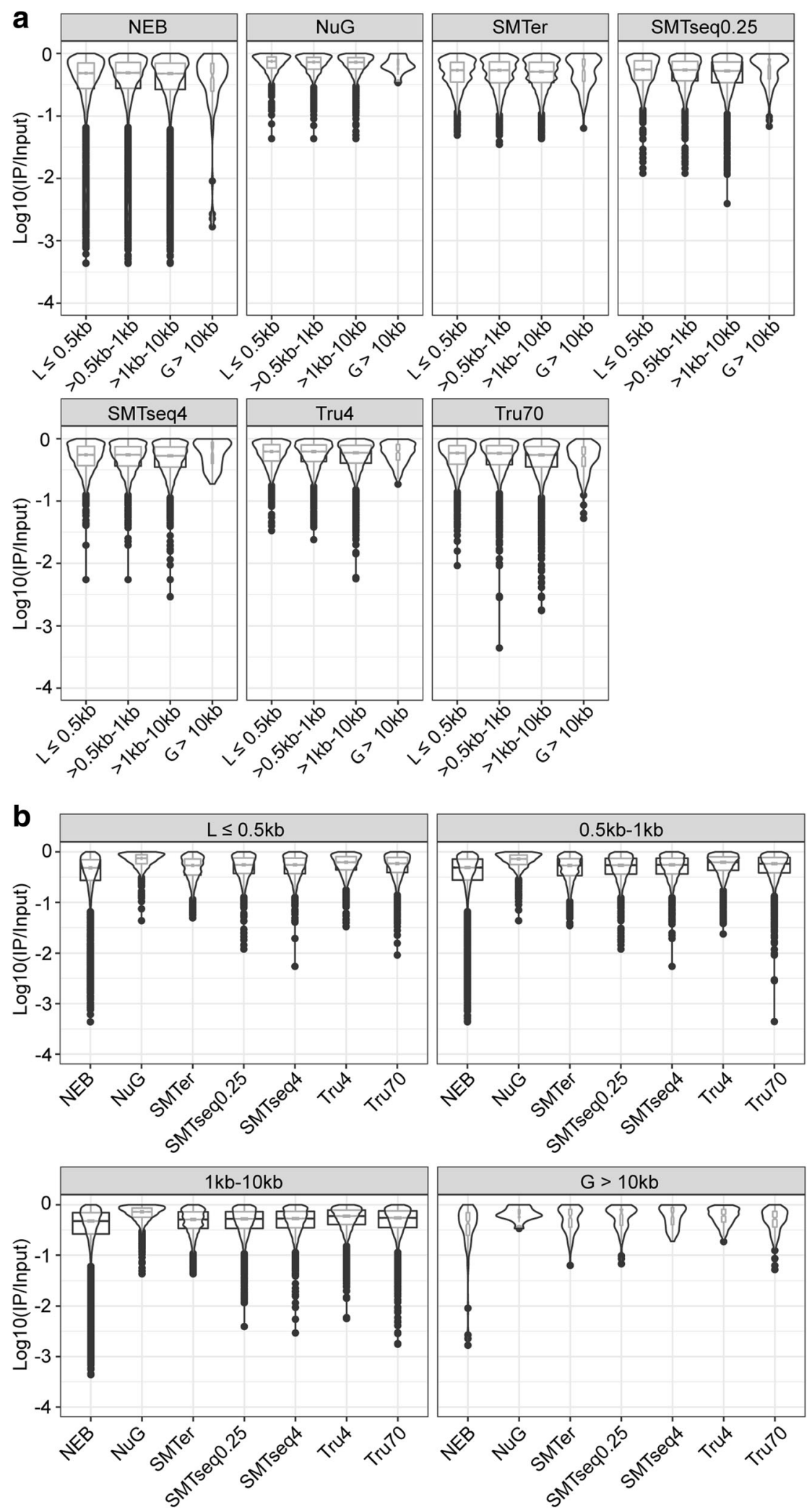

Fig. 10 Boxplot and violin plots of depletion features over different transcript lengths. a Boxplots of depletion factor of different transcripts within each kit. $\mathbf{b}$ Boxplots of depletion factor of different kits at each transcript length bin. The notch represents the median $\pm 1.58 \mathrm{IQR} / \mathrm{V} \mathrm{n}$. The width of boxplot is proportion to sample size of each group 
different tissues being used, and more specifically the percent of cells that express the tagged ribosomes. Among enriched transcripts, we observed an enrichment effect bias toward longer transcripts $(>10 \mathrm{~kb})$ (Fig. 9a). This may relate to the nature of RiboTag IP since it is a method to detect polysome profiles during translation [16]. It is possible that the higher number of ribosomes on longer transcripts leads to a higher enrichment. In addition, the greater enrichment effect of longer transcript is slightly higer for samples prepared by SMARTer and SMARTseq kits. This may be related to the template-switch oligonucleotide with one locked nucleic acid (LNA) technique applied in these kits, which is aimed to improve the hybridization between the template-switch oligonucleotide and the cDNA product [36, 37], increasing full coverage for longer transcripts.

\section{Conclusion}

Amongst the kits and library prep protocols analyzed in this manuscript, SMART-Seq v4 and TruSeq offer the best sequencing results for libray preparation from smaller amounts of RNA as starting material. Indeed, the overall profile of $250 \mathrm{pg} / 4 \mathrm{ng}$ samples from SMART-Seq v4 was similar to the TruSeq 70, here used as a gold-standard control. SMARTer Stranded Total RNA-Seq Kit might be a good choice to study both polyA $(+)$ mRNA and non-polyA mRNA, especially non-coding RNAs. Since there is a coverage bias towards $3^{\prime}$ for IP samples and more enrichment for longer transcripts, correction should be included during comparison among samples, for example, using the bias correction function in Cufflink [38]. Finally, IP RNA from RiboTag samples is likely to include a higher rate of immature RNAs, given the observed increase in intronic sequences in the IP samples across all library prep approaches. Overall, we were able to observe both enriched and depleted transcripts of translatome profiles using all kits. Greater enrichment effects were detected than depletion, however this may be related to the percent of tagged ribosomes in the tissue and therefore tissue and Cre-driver specific. In summary, by considering the eveness of coverage, number of detected features, low CPM of non-coding genes, and similar enrichment profiles comparing to standard TruSeq70 prepared samples, the SMARTseq and NEB kits performed the best in comparison to the other kits tested. However, the SMARTseq kit had a lower duplication rate and allows reactions to start with as little as $250 \mathrm{pg}$, significantly decreasing the necessary amount of starting material. In addition, the modified TruSeq4 protocol provides good results based on the relative high number of detected features, low $\mathrm{CPM}$ of non-coding genes, and similarity of the enrichment profile to the standard TruSeq70.

\section{Additional files}

Additional file 1: Table S1. Library preparation performed in this study. Table S2 Gene list with RPKM greater than 10,000. Table S3 Median of genes from unique and shared regions of Venn diagram. Table $\$ 4$ Spearman correlation coefficients between different profiles. (XLSX 28 kb)

Additional file 2: Figure S1. Cre recombination in liver cells expressing Gfi1. (a) Cryosection of liver from a cross between a Gfi1-Cre mouse and a TdTomato reporter mouse (Ai14) stained with DAPI and phalloidin. TdTomato is found in a subset of cells in the liver that is likely consistent with Kupffer or endothelial cells. (b) Enrichment of Gfil transcripts in the IP samples as compared to the input samples was assessed by reversetranscription followed by real time PCR. (PDF $2947 \mathrm{~kb}$ )

Additional file 3: Figure S2. Duplication rate of each library. X-axis: duplication rate, Y-axis: $\log 10$ of reads at different duplication rates. (PDF $622 \mathrm{~kb}$ )

Additional file 4: Figure S3. Hierarchical clustering of expression levels, based on the rank of the count of exon per million mapped reads (CPM). Dendrograms represent Spearman correlation coefficients between pairs of samples that is 3 replicates for input and 3 replicates for IP. (PDF $233 \mathrm{~kb}$ )

Additional file 5: Figure S4. Bland-Altman plot (MA plot) of translatome (IP samples) and transcriptome (input samples) profiles for each kit. The red lines represent the boundary cutoff $[-1,1]$. Dots above or below the red line represent the enriched or depleted transcripts. (JPG $587 \mathrm{~kb}$ )

Additional file 6: Figure S5. Venn diagram of enriched/depleted transcripts (CPM $\geq 20$ in at least one replicate, mean ratio of enrichement/ depletion of the three replicates). The mean ratio IP/input is $\geq 2$ or input/IP is $\geq 2$. (PDF $1063 \mathrm{~kb}$ )

Additional file 7: Figure S6. Histogram of length distribution for enriched or depleted transcripts. (PDF 353 kb)

\section{Abbreviations}

NEB: NEBNext ${ }^{\oplus}$ Ultra ${ }^{\text {TM }}$ Directional RNA Library Prep Kit for Illumina with 4 ng of RNA; NUGEN: NuGEN Ovation ${ }^{\oplus}$ RNA-Seq System V2 with 4 ng of RNA; SMARTer: TaKaRa SMARTer ${ }^{\circledast}$ Stranded Total RNA-Seq Kit-Pico Input Mammalian with $4 \mathrm{ng}$ of RNA; SMARTseq4 and SMARTseq0.25: TaKaRa SMART-Seq ${ }^{\bullet}$ v4 Ultra Low Input RNA Kit for Sequencing with 4 ng and 0.25 ng of RNA; TruSeq4 and TruSeq70: Illumina TruSeq RNA Library Prep Kit v2 with 4 ng and $70 \mathrm{ng}$ of RNA

\section{Acknowledgements}

We would like to thank Maggie Matern and Benjamin Shuster for critically reviewing this manuscript. This work was supported by R01DC03544 (NIDCD), R01DC013817 (NIDCD) and MR130240 (CDMRP/DoD).

\section{Funding}

This work was supported by R01DC03544 (NIDCD), R01DC013817 (NIDCD) and MR130240 (CDMRP/DoD). The funding sources provided the support for effort and materials, and did not have a role in study design, data collection and analysis, decision to publish or preparation of the manuscript.

\section{Availability of data and materials}

The datasets supporting the conclusions of this article are available in the NCBI Gene Expression Omnibus (GEO) repository under accession number GSE104213 https://www.ncbi.nlm.nih.gov/geo/query/acc.cgi?acc=GSE104213. The datasets supporting the conclusions of this article are included within the article and its additional files (Additional files 1, 2, 3, 4, 5, 6, and 7).

\section{Authors' contributions}

YS, BM, ETB, SO, XZ performed the experiments, YS, AS and AM performed the analysis, YS, BM and RH wrote the manuscript, AM, RJM, LT, LS and RH designed and oversaw the project. All the authors read and approved the final manuscript.

\section{Ethics approval and consent to participate}

All procedures involving animals were carried out in accordance with the National Institutes of Health Guide for the Care and Use of Laboratory 
Animals and have been approved by the Institutional Animal Care and Use Committee at the University of Maryland, Baltimore (protocol numbers 1,015,003 and 0915006).

\section{Consent for publication}

Not applicable.

\section{Competing interests}

The authors declare that they have no competing interests.

\section{Publisher's Note}

Springer Nature remains neutral with regard to jurisdictional claims in published maps and institutional affiliations.

\begin{abstract}
Author details
${ }^{1}$ Institute for Genome Sciences, University of Maryland School of Medicine, Baltimore, MD 21201, USA. ²Department of Otorhinolaryngology-Head \& Neck Surgery, University of Maryland School of Medicine, Baltimore, MD 21201, USA. ${ }^{3}$ Genomics and Computational Biology Core, National Institute on Deafness and Other Communication Disorders, Bethesda, MD 20892, USA. ${ }^{4}$ Department of Anatomy and Neurobiology, University of Maryland School of Medicine, Baltimore, MD 21201, USA.
\end{abstract}

\section{Received: 10 November 2017 Accepted: 11 September 2018}

\section{Published online: 21 September 2018}

\section{References}

1. JM S: Using fluorescence activated cell sorting to examine cell-type-specific gene expression in rat brain tissue. J Vis Exp 2015, 99:e52537.

2. Jung J, Jung $\mathrm{H}$. Methods to analyze cell type-specific gene expression profiles from heterogeneous cell populations. Animal Cells and Systems. 2016;20:113-7.

3. Kim T, Lim CS, Kaang BK. Cell type-specific gene expression profiling in brain tissue: comparison between TRAP, LCM and RNA-seq. BMB Rep. 2015; 48(7):388-94.

4. Chen L, Borozan I, Sun J, Guindi M, Fischer S, Feld J, Anand N, Heathcote J, Edwards AM, McGilvray ID. Cell-type specific gene expression signature in liver underlies response to interferon therapy in chronic hepatitis $C$ infection. Gastroenterology. 2010;138(3):1123-33 e1121-1123.

5. Li J, Klughammer J, Farlik M, Penz T, Spittler A, Barbieux C, Berishvili E, Bock C, Kubicek S. Single-cell transcriptomes reveal characteristic features of human pancreatic islet cell types. EMBO Rep. 2016;17(2):178-87.

6. Li M, Jia C, Kazmierkiewicz KL, Bowman AS, Tian L, Liu Y, Gupta NA, Gudiseva HV, Yee SS, Kim M, et al. Comprehensive analysis of gene expression in human retina and supporting tissues. Hum Mol Genet. 2014;23(15):4001-14

7. Hertzano R, Elkon R, Kurima K, Morrisson A, Chan SL, Sallin M, Biedlingmaier A, Darling DS, Griffith AJ, Eisenman DJ, et al. Cell type-specific transcriptome analysis reveals a major role for Zeb1 and miR-200b in mouse inner ear morphogenesis. PLoS Genet. 2011;7(9):e1002309.

8. Cheng L, Zhang S, MacLennan GT, Williamson SR, Davidson DD, Wang M, Jones TD, Lopez-Beltran A, Montironi R. Laser-assisted microdissection in translational research: theory, technical considerations, and future applications. Appl Immunohistochem Mol Morphol. 2013;21(1):31-47.

9. Asano T, Masumura T, Kusano H, Kikuchi S, Kurita A, Shimada H, Kadowaki K Construction of a specialized cDNA library from plant cells isolated by laser capture microdissection: toward comprehensive analysis of the genes expressed in the rice phloem. Plant J. 2002;32(3):401-8.

10. Smalley MJ, Titley J, O'Hare MJ. Clonal characterization of mouse mammary luminal epithelial and myoepithelial cells separated by fluorescenceactivated cell sorting. In Vitro Cell Dev Biol Anim. 1998;34(9):711-21.

11. Birnbaum K, Shasha DE, Wang JY, Jung JW, Lambert GM, Galbraith DW, Benfey PN. A gene expression map of the Arabidopsis root. Science. 2003; 302(5652):1956-60.

12. Graham M, Richardson JL, Macara IG. Does facs change gene expression. cytometry. 2015;87:166-75.

13. Streets AM, Huang Y. How deep is enough in single-cell RNA-seq? Nat Biotechnol. 2014;32(10):1005-6.

14. Wu AR, Neff NF, Kalisky T, Dalerba P, Treutlein B, Rothenberg ME, Mburu FM, Mantalas GL, Sim S, Clarke MF, et al. Quantitative assessment of single-cell RNA-sequencing methods. Nat Methods. 2014;11(1):41-6.
15. Doyle JP, Dougherty JD, Heiman M, Schmidt EF, Stevens TR, Ma G, Bupp S, Shrestha P, Shah RD, Doughty ML, et al. Application of a translational profiling approach for the comparative analysis of CNS cell types. Cell. 2008; 135(4):749-62.

16. Sanz E, Yang L, Su T, Morris DR, McKnight GS, Amieux PS. Cell-type-specific isolation of ribosome-associated mRNA from complex tissues. Proc Natl Acad Sci U S A. 2009;106(33):13939-44.

17. Heiman M, Schaefer A, Gong S, Peterson JD, Day M, Ramsey KE, SuarezFarinas M, Schwarz C, Stephan DA, Surmeier DJ, et al. A translational profiling approach for the molecular characterization of CNS cell types. Cell. 2008;135(4):738-48.

18. Brar GA, Weissman JS. Ribosome profiling reveals the what, when, where and how of protein synthesis. Nat Rev Mol Cell Biol. 2015;16(11):651-64.

19. Shigeoka T, Jung H, Jung J, Turner-Bridger B, Ohk J, Lin JQ, Amieux PS, Holt CE. Dynamic axonal translation in developing and mature visual circuits. Cell. 2016;166(1):181-92.

20. Levin JZ, Yassour M, Adiconis X, Nusbaum C, Thompson DA, Friedman N, Gnirke A, Regev A. Comprehensive comparative analysis of strand-specific RNA sequencing methods. Nat Methods. 2010;7(9):709-15.

21. Faherty SL, Campbell CR, Larsen PA, Yoder AD. Evaluating whole transcriptome amplification for gene profiling experiments using RNA-Seq BMC Biotechnol. 2015;15:65.

22. Shanker S, Paulson A, Edenberg HJ, Peak A, Perera A, Alekseyev YO, Beckloff N, Bivens NJ, Donnelly R, Gillaspy AF, et al. Evaluation of commercially available RNA amplification kits for RNA sequencing using very low input amounts of total RNA.J Biomol Tech. 2015:26(1):4-18.

23. Adiconis X, Borges-Rivera D, Satija R, DeLuca DS, Busby MA, Berlin AM, Sivachenko A, Thompson DA, Wysoker A, Fennell T, et al. Comparative analysis of RNA sequencing methods for degraded or low-input samples. Nat Methods. 2013:10(7):623-9.

24. Matern M, Vijayakumar S, Margulies Z, Milon B, Song Y, Elkon R, Zhang X, Jones SM, Hertzano R. Gfi1Cre mice have early onset progressive hearing loss and induce recombination in numerous inner ear non-hair cells. Sci Rep. 2017;7:42079

25. Pearson WR, Wood T, Zhang Z, Miller W. Comparison of DNA sequences with protein sequences. Genomics. 1997;46(1):24-36.

26. Orvis J, Crabtree J, Galens K, Gussman A, Inman JM, Lee E, Nampally S, Riley $D$, Sundaram JP, Felix $V$, et al. Ergatis: a web interface and scalable software system for bioinformatics workflows. Bioinformatics. 2010;26(12):1488-92.

27. Kim D, Pertea G, Trapnell C, Pimentel H, Kelley R, Salzberg SL. TopHat2: accurate alignment of transcriptomes in the presence of insertions, deletions and gene fusions. Genome Biol. 2013;14(4):R36.

28. Anders $\mathrm{S}, \mathrm{Huber} W$. Differential expression analysis for sequence count data Genome Biol. 2010;11(10):R106.

29. Quinlan AR, Hall IM. BEDTools: a flexible suite of utilities for comparing genomic features. Bioinformatics. 2010;26(6):841-2.

30. Mortazavi A, Williams BA, McCue K, Schaeffer L, Wold B. Mapping and quantifying mammalian transcriptomes by RNA-Seq. Nat Methods. 2008; 5(7):621-8.

31. Krzywinski M, Altman N. Visualizing samples with box plots. Nat Methods. 2014;11(2):119-20.

32. Yang H, Gan J, Xie X, Deng M, Feng L, Chen X, Gao Z, Gan L. Gfi1-Cre knock-in mouse line: a tool for inner ear hair cell-specific gene deletion. Genesis. 2010;48(6):400-6.

33. Parekh S, Ziegenhain C, Vieth B, Enard W, Hellmann I. The impact of amplification on differential expression analyses by RNA-seq. Sci Rep. 2016;6:25533.

34. Combs PA, Eisen MB. Low-cost, low-input RNA-seg protocols perform nearly as well as high-input protocols. PeerJ. 2015;3:e869.

35. Roh HC, Tsai LT, Lyubetskaya A, Tenen D, Kumari M, Rosen ED. Simultaneous transcriptional and Epigenomic profiling from specific cell types within heterogeneous tissues in vivo. Cell Rep. 2017;18(4):1048-61.

36. Picelli S, Bjorklund AK, Faridani OR, Sagasser S, Winberg G, Sandberg R. Smart-seq2 for sensitive full-length transcriptome profiling in single cells. Nat Methods. 2013;10(11):1096-8.

37. Picelli S, Faridani OR, Bjorklund AK, Winberg G, Sagasser S, Sandberg R. Fulllength RNA-seq from single cells using Smart-seq2. Nat Protoc. 2014;9(1): 171-81.

38. Trapnell C, Williams BA, Pertea G, Mortazavi A, Kwan G, van Baren MJ, Salzberg SL, Wold BJ, Pachter L. Transcript assembly and quantification by RNA-Seq reveals unannotated transcripts and isoform switching during cell differentiation. Nat Biotechnol. 2010;28(5):511-5. 